\title{
Integrative proteomic and metabolomic analysis reveals the cotton plant defense mechanisms induced by insect (Adelphocoris suturalis Jakovlev) feeding
}

Hui Lu ${ }^{1,2,3}$, Shuai Zhang ${ }^{1}$, JunYu Luo ${ }^{1}$, ChunYi Wang ${ }^{1}$, LiMin Lv ${ }^{1}$, LiJuan Zhang ${ }^{1}$, XiangZhen Zhu ${ }^{1}$, Li Wang ${ }^{1}$, XueKe Gao ${ }^{1}$, HongXia Hua ${ }^{2}$, JinJie Cui ${ }^{1 *}$ 7 8

${ }^{1}$ State Key Laboratory of Cotton Biology, Institute of Cotton Research of CAAS, Anyang 455000, China.

${ }^{2}$ Hubei Insect Resources Utilization and Sustainable Pest Management Key Laboratory, College of Plant Science and Technology, Huazhong Agricultural University, Wuhan, 430070, China.

${ }^{3}$ Hetao college, Inner Mongolia, 015000, China.

${ }^{*}$ Corresponding Author:

Dr. JinJie Cui

Plant Protection Department, Institute of Cotton Research, Chinese Academy of Agricultural

Sciences, No. 38, Huanghe Road, Anyang 455000, Henan, People's Republic of China.

Tel/Fax: 0086-0372-2562296

Email: aycuijinjie@163.com 


\section{Abstract}

44 Cotton (Gossypium hirsutum Linn.) is widely cultivated in China. The polyphagous insect 45 Adelphocoris suturalis (Jakovlev) is a serious insect pest in cotton growing regions. Plants have evolved sophisticated systems to cope with herbivore attacks. However, the cotton defense mechanisms induced by A. suturalis feeding have lagged behind. We carried out untargeted proteomic analysis using the iTARQ technique and metabolomics based on LC-MS/MS analysis of cotton leaves fed upon by $A$. suturalis. Proteomic analysis identified 775 upregulated proteins and 477 downregulated proteins in plants that were infested by A. sututralis compared to the controls. Metabolomic analysis identified 50 differentially expressed metabolites in the positive ion mode and 14 in the negative ion mode compared to the controls. The tryptophan metabolism pathway was significantly changed in both the positive and negative ion mode in the metabolomics analysis. The alpha-linolenic acid pathway was significantly changed in both the proteomic and metabolomics analyses. Furthermore, the result was validated by RT-qPCR analysis of 5 related genes involved in alpha-linolenic acid pathway. These results indicate that tryptophan metabolism and the alpha-linolenic acid pathway may be important in cotton defense against herbivores and would enhance our understanding of plant defenses induced by A. sututrali feeding.

60 


\section{Introduction}

Plants have successfully colonized most environments where herbivores are common because they have evolved sophisticated systems to cope with herbivore feeding [1]. Some plants can release volatile organic chemicals (VOCs) after herbivore infestation [2], while other plants have developed structural defenses, such as spines, trichomes, and thick, tough leaves [3]. When plants receive physical and chemical cues from herbivorous insects, such as elicitors in oral secretions and compounds in oviposition fluids, they can alter their proteins and metabolites. Plant defense responses induced by herbivores can occur in both wounded and in undamaged regions [4]. Herbivores, in turn, have responded to plant defenses by evolving counter adaptations that make plant defenses less effective or render them useless. This can lead to an evolutionary "arms race", between plants and their herbivore enemies [5,6].

Cotton (G. hirsutum) is an important cash crop worldwide. In China, the development and widespread adoption of transgenic Bt (Bacillus thuringiensis) cotton has led to a substantial reduction in the use of broad-spectrum insecticides. This in turn has led to frequent outbreaks of mirids [7-9]. Two species in the Miridae, Adelphocoris suturalis and Apolygus lucorum, are emerging as the most destructive pests in the major cotton growing regions. These species are highly polyphagous and attack a broad range of cultivated crops, such as cotton, beans, alfalfa, vegetables, and fruit crops [10]. Both the nymphs and adults of these species suck plant sap from cotton flower buds, tender shoots, and buds, resulting in abscission, wilting, abnormal growth, and losses in lint yield and quality [11].

The detection and quantification of multiple proteins and metabolites can be accomplished using techniques such as LC-Q/TOF-MS-based metabolomics [12-15]. Quantitative iTRAQ-LCMS/MS proteomics can be a sensitive method for high-throughput protein identification and quantification [16]. It has been used in insects and plants, including the silkworm[17], brown planthopper[18], pine beetle[19], locust[20], and cotton [21,22]. These techniques can provide a global view of dynamic proteomic and metabolomic variation and allow for the discovery of key proteins and metabolites that are essential for plant defenses. However, there have been few reports on cotton proteins and chemical metabolite changes after attacks by A. suturalis. In this study, we used a proteomic and metabolomic approach to study cotton plant defenses in response to A. suturalis feeding. Tryptophan metabolism and alpha-linolenic acid metabolism are important pathways that regulate plant development and defense responses induced by pathogens and insects [23-26]. We evaluate the new data and discuss how the tryptophan metabolism and alpha-linolenic acid pathways play essential roles in cotton defenses. The present study provides a new insight into the molecular mechanism of plant defense induced by insect feeding.

\section{Results}

2.1. iTRAQ identified the different proteins in the cotton treatment with A. suturalis infested for $48 \mathrm{~h}$ and the control without insect pressure

Cotton proteomic analysis by iTRAQ was performed on three replicates as previously described [21,22,27-29]. A total of 371575,374246 , and 373127 spectra were generated in the treatments of P48E, and the two controls of P48C and P0C. P48E represents plants infested with insects for $48 \mathrm{~h}$; $\mathrm{P} 48 \mathrm{C}$ represents plants grown for $48 \mathrm{~h}$ without insect infestation; and P0C represents experimental cotton plants without insect infestation. We obtained 52750, 48103, and 52474 unique spectra, 17865, 16116, and 16483 identified peptides, including 12906, 11903, and 12135 unique peptides and 5520, 5210, and 5313 identified proteins from the cotton in P48E, 
$134 \mathrm{P} 48 \mathrm{C}$, and P0C, respectively, with a false discovery rate (FDR) of $<1 \%$ (Table S1). And finally, a 135 total of 8302 proteins were identified (Table S1).

136 2.2. Functional categories of differentially expressed proteins

137

138

139

140

141

142

143

144

145

146

147

148

149

150

151

152

153

154

155

156

157

158

159

160

161

162

163

164

165

166

167

168

169

170

171

172

173

174

175

176

177

178

179

In this study, any proteins with $\mathrm{a} \geq 1.2$-fold difference and a $q$-value $<0.05$ were designated as significant differently expressed proteins (DEPs) [30-34]. We got $q$-value through $p$-value corrected for false discovery rate (FDR) by Benjamini-Hochberg (BH). A total of $1015(56 \%)$ proteins were upregulated and 796 (44\%) were downregulated in P48E compared to the control P0C. A total of 775 (62\%) proteins were upregulated and 477 (38\%) were downregulated in P48E compared to the control P48C. There were 485 (48\%) proteins that were upregulated and 520 (52\%) were downregulated in P48C compared to the control P0C (Fig. 1A, Table S2). Venn diagram shows common or uniquely up- and down-regulated proteins in different experimental groups. (Fig. 1B, 1C). The remaining pathway enrichment categories are shown in Table 1 . The results suggest that plants can alter their proteins when attacked by herbivorous insects, and then plant defense responses are induced.

To study the functional categories of the insect feeding treatment (P48E) and its control (P48C), we conducted GO enrichment analysis of DEPs. DEPs were classified into three groups: biological process, cellular component, and molecular function (Fig. 1D). For biological process, the proteins were predominantly distributed in the metabolic process (258), single-organism process (225), cellular process (58), response to stimulus (45), and localization (38). For cellular components, the proteins were predominantly distributed in the organelle part (98), organelle (90), cell part (83), membrane part (46), and macromolecular complex (27). For molecular function, the proteins were predominantly distributed in the catalytic activity (527), binding (125), transporter activity (31), structural molecule activity (29), and antioxidant activity (26) (Fig. 1D).

For analysis of the metabolic pathways of the cotton plants infested by A. suturalis, DEPs were also investigated using the KEGG database (ver. 81). We first compared the two control groups, P48C and P0C (Table S3), and then removed the pathway changes arising from plant growth. These changes were considered as background noise. Then, we compared experimental $\mathrm{P} 48 \mathrm{E}$ with the control P48C, and found that the DEPs were enriched in alpha-linolenic acid metabolism (1.85\%), fructose and mannose metabolism (2.53\%), amino sugar and nucleotide sugar metabolism $(4.09 \%)$, selenocompound metabolism (1.17\%), protein digestion and absorption $(0.49 \%)$. The remaining pathway enrichment categories are shown in Table 1 . Those results indicated that metabolic pathway may play an important role in plant defense.

2.3. Metabolome changes in cotton plant in response to $A$. suturalis feeding

Reproducibility of the UPLC-Q-TOF-MS was determined from ten replicates with the same quality control (QC) sample interspersed throughout the analysis (Fig. 2A). In all of the QC samples, ions with relative standard deviation (RSD) $>30 \%$ were deleted. We then had 7513 positive ions ( $\mathrm{RSD} \leq 30 \%, 96.44 \%$ ) of the total 7790 , and 4053 negative ions (RSD $\leq 30 \%$, $93.71 \%$ ) of the total 4325 . To investigate cotton metabolic changes in response to A. suturalis feeding, all of the observations, acquired in both positive and negative ion modes, were analyzed using two components principal component analysis (PCA) score (Fig. 2B). To best analyze the metabolic variations of the $A$. suturalis feeding groups, all of the observations acquired in both ion modes were analyzed using orthogonal partial least squares-discriminant analysis (OPLS-DA). The differential metabolites were selected according to the variable important for the projection (VIP) threshold (VIP > 1) in the OPLS-DA model with the $q$-value $(q<0.05)$ after FDR correction [32-34]. The plots of the OPLS-DA model discriminated the insect feeding groups from their corresponding control groups, and exhibited satisfactory classification (Fig. 2C). Under this 
180 standard, there were 70 (18 upregulated and 52 downregulated) metabolites in the positive ion 181 mode and 15 (all downregulated) in the negative ion mode (Table S4). A clear metabolite 182 separation was observed between the A. suturalis feeding groups and the corresponding control 183 groups as illustrated in a heat-map (Fig. 3, Table S4). The results suggest that metabolites were downregulated and this may induce cotton' defense against herbivorous insects.

To further analyze the cotton metabolic pathways, different metabolites were studied using the KEGG database. Comparing the insect feeding group with the control group, in positive ion mode, the top 6 pathways were metabolic pathways, biosynthesis of secondary metabolites, sesquiterpenoid and triterpenoid biosynthesis, tryptophan metabolism, isoquinoline alkaloid biosynthesis, tropane, piperidine and pyridine alkaloid biosynthesis. In the negative ion mode, the top 6 pathways were metabolic pathways, biosynthesis of secondary metabolites, alpha-linolenic acid metabolism, tryptophan metabolism, phenylpropanoid biosynthesis, isoquinoline alkaloid biosynthesis. The metabolites in metabolic pathways, biosynthesis of secondary metabolites, alpha-linolenic acid metabolism and tryptophan metabolism were significantly changed in both positive and negative ion modes (Table 2).

\subsection{Tryptophan metabolism in cotton after feeding by $A$. suturalis}

We integrated positive and negative ion mode data to analyze the tryptophan metabolism pathway in the treatment cotton feeding by A. suturalis compared to the control cotton. We found that there were 21 metabolites including 18 metabolites in positive ion mode and 3 metabolites in negative ion mode were downregulated compared to the control group; while only 4 metabolites in positive ion mode were upregulated compared to the control group; and there was 1 metabolite in negative ion mode was downregulated but it was upregulated in positive ion mode (Fig. 4A; Table 3 ). The results showed that among 26 changed metabolites in tryptophan metabolism pathway, 21 $(80.8 \%)$ metabolites were downregulated and only 4 (15.4\%) metabolites were upregulated, these results indicated that most of metabolites in tryptophan metabolism pathway were downregulated and this may be an important response for cotton to defend against herbivores, the results described here in Fig. 4B.

2.5. Integrating proteomics and metabolomics data to analyses alpha-linolenic acid metabolism pathway

Interestingly, we found that the alpha-linolenic acid metabolism pathway was significantly changed in both the proteomic and metabolomic analyses (Table 1,2). We investigated the pathway enrichment of P48E vs P48C in both omics. Most of the metabolites were downregulated (Fig. 5, blue rectangular box), except for volicitin and 3-hexenol (Fig. 5, red rectangular box), while most related proteins were upregulated (Fig. 5, red elliptic box). These proteins included allene oxide synthase (AOS), cytochrome P450 allene oxide synthase (CytP450), phospholipase A (PLA), alcohol dehydrogenase (ADH), allene oxide cyclase 4 (AOC4), 12-oxophytodienoate reductase (OPR), AMP-dependent CoA ligase, acyl-CoA oxidase 4 (ACO4), acyl-CoA oxidase 3 (ACO3), and 3-ketoacyl-CoA thiolase 2(3-KAT-2) (Table S5). This finding indicated proteins as the upstream regulator increased led to the downstream metabolites deceased in alpha-linolenic acid metabolism pathway. The reconfiguration of proteins and metabolites may be one of the ways that plants cope with insect-feeding stress. There were several unknown proteins that may also play important roles in alpha-linolenic acid metabolism (Fig. 5). We selected 5 proteins including AOS, PLA1, AOC4, and two OPR proteins named OPR1 and OPR2, which were related to the alpha-linolenic acid metabolism pathway (Table S5), and performed quantitative reverse transcription PCR (RT-qPCR) analysis. Two housekeeping genes [35] (GhHis3 with GenBank 
accession AF024716 and GhUBQ7 with GenBank accession DQ116441) were used. The RTqPCR analysis validated our sequencing results (Fig. 6).

\section{Discussion}

Plants have evolved sophisticated systems to cope with herbivore attacks. When plants perceive herbivore-derived physical and chemical cues, they can dramatically reshape their transcriptomes, proteomes, and metabolomes. These responses involve specific changes in metabolism, gene expression, and in the pattern of plant growth and development[36-38].

Secondary metabolic compounds of plants are an important biochemical basis for plant resistance to insects [39]. Research with many plant species has revealed a great variety of small molecules with toxic or antifeedant effects on insect herbivores. For example, many terpenoids, the most metabolically diverse class of plant secondary metabolites, play important roles in plant defenses. Alkaloids (e.g., caffeine, nicotine, morphine, strychnine, and cocaine) are also secondary plant metabolites that help protect plants from herbivores [40]. Other well-studied classes of plant secondary metabolites with defensive properties include furanocoumarins, cardenolides, tannins, saponins, glucosinolates, and cyanogenic glycosides [41,42]. Cotton plants, coping with the stress from insect feeding, have evolved numerous inducible defense mechanisms that help them respond to biotic stress, including the synthesis of volatile terpenes, phytoalexins, gossypol, tannins, tyloses, pathogenesis-related proteins, as well as lignification, and the release of active oxygen species [39,42-45].

Tryptophan is the amino acid metabolic precursor of many important secondary metabolites. Tryptophan biosynthesis plays a direct role in regulating plant development, pathogen defense responses, and plant-insect interactions [25,44-47]. In plants, several important secondary metabolites are derived from tryptophan or its indolic precursors. These include the plant growth regulator indole-3-acetic acid (IAA) and the pathogen defense compounds indole glucosinolates (IGs) and indolic phytoalexins [45]. In the present study, we examined the molecular responses of cotton to insect feeding stress using metabolomics based on LC-MS/MS analysis. Among changed metabolites associated with the tryptophan metabolism pathway we found that there were 5 upregulated and 22 downregulated. Tryptophan metabolism is mainly downregulated and this may promote the effectiveness of the plant immune system.

Jasmonate (JA) modulates numerous physiological processes that are related to plant development and defense responses [24,25]. Oxygenation of alpha-linolenic acid is the initial step in JA biosynthesis [25]. Alpha-linolenic acid is a stress signal released by lipase activity on chloroplast membranes. It is the substrate for numerous oxygenated compounds collectively called oxylipins, including JA, which comprise JA, MeJA (methyl jasmonate), JA amino acid conjugates, and further JA metabolites [4,24]. It can be released into the plastid under stress conditions [4,24]. The related proteins in the alpha-linolenic acid metabolism pathway AOS, AOCs (AOC1, 2, 3, and 4), and OPR3(12-Oxophytodienoate reductase 3) are key protiens involved in the synthesis of JA. AOSs belong to the CYP74A enzyme family [48]. The AOC is the subsequent enzyme of the AOS branch [49]. OPR3 belong to a family of flavoproteins identified first with Warburg's old yellow enzyme (OYE), and then from 13-hydroperoxylinolenic acid (13-HPOTrE) [24]. This fatty acid hydroperoxide is then dehydrated by AOS and cyclized by AOC to the cyclopentenone 12oxo-phytodienoic acid (12-OPDA) [24]. OPDA is a potent gene regulator in the wound response and can protect plants against the attack of insect or fungal pathogens when JA is absent [50]. In our present study, we integrated proteomic and metabolomic data of the alpha-linolenic acid metabolism pathways and we found that proteins, such as AOS, AOC4, OPR1, OPR2, were upregulated, and this is similar to the result which were found in the rice feeding by stem borers 
271 [51], while in metabolites, such as 13(s)-HpOTrE,9,10-EoTrE, colnelenic acid, O-OPDA,12-

272 OPDA, and 9(s)-HpOTrE, were downregulated. This result indicated that proteins as the upstream regulators increased and led to a decrease in downstream metabolites. Volicitin (N-(17hydroxylinolenoyl)-L-glutamine) was first isolated from oral secretions of beet armyworm caterpillars [52]. The linolenic acid derivative volicitin induces maize (Zea mays L.) seedlings to release volatile compounds (terpenoids and indole) that are similar to those released from plants damaged by caterpillar feeding [52]. And in our present result, volicitin were upregulated in the alpha-linolenic acid pathway after feeding by $A$. suturalis, this suggest that volicitin may function the cotton defense.

We chose 5 proteins and performed RT-qPCR, and we found the expression of those genes validated our sequencing results. At the beginning of the insect infested time (3-6h), all of the genes are at a low expression level, and then at $24 \mathrm{~h}$ and $48 \mathrm{~h}$, there is a peak expression level, after that, the expression begin to decrease (Fig.6). This result suggest that plant can regulate their proteins to cope with insect feeding. Finally, a better understanding of why the plant reshapes its proteins and metabolites after insect attack is important to explain the relationship between the plant and the insect. Future research should focus on the unknown proteins in the alpha-linolenic acid metabolism pathway.

\section{Materials and Methods}

\subsection{Insect rearing}

A. suturalis were reared at $26^{\circ} \mathrm{C}, 75 \pm 5 \%$ relative humidity, with a photoperiod of $16: 8 \mathrm{~h}$ (L:D) in cages (30x30x30 cm, 1000 nymphs/cage). Insects were fed green beans, mung bean seeding, and cotton bollworm (Helicoverpa armigera Hübner) eggs [53].

4.2. Plant Material and insect infestation

Potted cotton plants ( $G$. hirsutum) were soil grown in controlled-environment chambers under a regime of either a 10 -h (short-day) or 16-h (long-day) light period at $25^{\circ} \mathrm{C}$ and $65 \%$ relative humidity, unless otherwise indicated. After 3 weeks of growth, cotton plant was transferred into one $30-\mathrm{cm}$ square cage, and 9 adult bugs were released on the cotton leaves. Our preliminary experiment indicated that $24 \mathrm{~h}$ and $48 \mathrm{~h}$ were the optimal termination time and this result was similar to the rice feeding by stem borers [51]. After $48 \mathrm{~h}$ of insect infestation, all of the insects were removed. The leaves were immediately frozen in liquid nitrogen and stored at $-80^{\circ} \mathrm{C}$ until use. For proteomic sequencing, each treatment had three experimental replicates, with $\mathrm{P} 48 \mathrm{E}$ representing the plants that were infested with insects for $48 \mathrm{~h}$. The P48C control plants were not infested but simply grown for $48 \mathrm{~h}$. The P0C controls were the experimental cotton plants without insect infestation. And for RT-qPCR analysis, we selected 5 proteins and performed 6 experiments, P3E, P6E, P12E, P24E, P48E and P72E, which represent 3 h, 6 h, 12 h, 24 h, 48 h, and $72 \mathrm{~h}$ of insect infestation, respectively. The control plants were not infested but simply grown for $3 \mathrm{~h}, 6$ h, $12 \mathrm{~h}, 24 \mathrm{~h}, 48 \mathrm{~h}$, and $72 \mathrm{~h}$, respectively. The main leaf and cotyledon were immediately frozen in liquid nitrogen, respectively, and stored at $-80^{\circ} \mathrm{C}$ until use. For metabolomic sequencing, each treatment had ten experimental replicates, with group 1 representing plants that were infested with insects for $48 \mathrm{~h}$ and group 2 representing plants without insect infestation but grown for $48 \mathrm{~h}$ as the controls. 4.3. Protein preparation and iTRAQ labeling

The iTRAQ analysis was conducted at BGI (Shenzhen, China). Total proteins were extracted from cotton plants using a previously reported phenol extraction procedure [54]. Protein concentrations were determined using the Bradford method [55]. Three independent biological replicates were performed in the experiment. A total of $25 \mu \mathrm{g}$ total protein from each sample was 
317 used for each experiment. Protein was digested by sequencing grade trypsin (Promega) at a ratio 318 of 1:10 (w:w) for $12 \mathrm{~h}$ at $37^{\circ} \mathrm{C}$, and then labeled using iTRAQ 8-plex kits (AB Sciex Inc., MA, 319 USA) according to the manufacturer's instructions. The samples were labeled with iTRAQ tags 320113 (P48E, $48 \mathrm{~h}$ experiment), 119 (P48C, $48 \mathrm{~h} \mathrm{CK}$ ), and 121 (P0C, 0 h CK), respectively.

\subsection{LC-MS/MS analysis}

LC-MS/MS analysis was performed as described previously [21,22]. After labeling all of the samples mixing, HPLC separation, and LC-MS/MS analysis. For MS, balance group can show the same $\mathrm{m} / \mathrm{z}$ no matter which report ion label peptide. In MS2, neutral loss happened to the balance group, the intensity of the report ion can reflect the relative abundance of the peptides. Data was collected with the AB SCIEX Triple TOF 5600 System (Concord, USA) fitted with a Nanospray III source (Concord, USA) with a pulled quartz tip as the emitter (New Objectives, Woburn, USA). The MS was operated with an RP greater than or equal to 30,000 FWHM for TOF MS scans.

\section{5. iTRAQ protein identification and quantification}

Protein identification and quantification were performed with the Mascot 2.3.02 search engine (Matrix Science, Boston, MA). The protein mass is predicted by website (http:// www.expasy.ch/tools/) based on the protein sequences. Search settings were used as described previously [22]. The Searches were made against database from the Institute of cotton research of Chinese Academy of Agriculture Science website

(http://cgp.genomics.org.cn/page/species/index.jsp).The search parameters were in Table S6. To demonstrate the reproducibility of the replicates, protein abundances between various biological replicates were compared, and ratios for each protein in each comparison were normalized to 1 . For quantitative changes, a 1.2-fold cutoff was set to determine upregulated and downregulated significant proteins, with q-value $<0.05$ (FDR corrected by BH) present in at least one replicate [30-34].

The mass spectrometry proteomics data were deposited in the ProteomeXchange Consortium (http://proteomecentral.proteom exchange.org) via the PRIDE partner repository [56] with the dataset identifier PXD007518. The ProteomeXchange reviewer account: reviewer77720@ebi.ac.uk password:DzF4ptow. 4.6. Bioinformatic analysis of proteins

Functional annotation of proteins was conducted using the Blast2GO program against the non-redundant protein database (NR; NCBI). The KEGG database (http://www. genome.jp/kegg/) and the Clusters of Orthologous Groups (COG) database (http://www. ncbi.nlm.nih.gov/COG/) were used to classify and group identified proteins. GO and pathway enrichment analysis were performed to determine which functional subcategories and metabolic pathways were overrepresented by the differentially accumulated proteins.

\subsection{LC-MS-based metabolomics}

The LC-MS-based targeted metabolomic analysis was performed according to a previously described protocol [34,51]. Unbiased metabolomic profiles of cotton samples were obtained using HPLC-MS. All of the cotton samples were rapidly flash-frozen in liquid nitrogen and stored at $-80^{\circ} \mathrm{C}$ until processing. A $25-\mathrm{mg}$ sample was ground in liquid nitrogen, and transferred into a 1.5$\mathrm{ml}$ polypropylene tube. Then, $800 \mu \mathrm{L}$ of chilled methanol/water $(1: 1)$ buffer solution, and two small balls were added to each tube. A tissue Lyser was set at the frequency of $60 \mathrm{~Hz}$ and the tube contents were shaken for $5 \mathrm{~min}$ followed by centrifugation at $25000 \mathrm{rpm}$ for $10 \mathrm{~min}$ at $4{ }^{\circ} \mathrm{C}$. We used QC samples to assess the reproducibility and reliability of the LC-MS system. Each tube was centrifuged with $200 \mu \mathrm{L}$ supernatant (sample code + up) and we then centrifuged another $200 \mu \mathrm{L}$ 
363 supernatant mixed for QC mark. We added $200 \mu \mathrm{L}$ supernatant to each tube as a QC sample and

364 then dried it via vacuum freezing. We removed all of the supernatant and dried precipitate, then

365 added $800 \mu \mathrm{L}$ ice-cold mixture of dichloromethane/methanol (3:1) The tissue Lyser was set at a

366 frequency of $60 \mathrm{~Hz}$, shaken for $5 \mathrm{~min}$, and the mixture was centrifuged at $25000 \mathrm{rpm}$ for $10 \mathrm{~min}$ at

$3674^{\circ} \mathrm{C}$. Each tube centrifuged $200 \mu \mathrm{L}$ supernatant (sample code + down) and then centrifuged another

$368200 \mu \mathrm{L}$ supernatant mixed for QC mark. Each tube had $200 \mu \mathrm{L}$ supernatant added as the QC

369 sample and then dried by vacuum freezing. Samples-up and samples-down were dissolved with

$37050 \%$ methanol, shaken for $1 \mathrm{~min}$, centrifuged at $25000 \mathrm{rpm}$ for $10 \mathrm{~min}$ at $4^{\circ} \mathrm{C}$. We added $100 \mu \mathrm{L}$

371 supernatant to 96 -well plates.

372

373

374

375

376

377

378

379

380

Sample analysis was conducted in both positive electrospray ionization (ESI+) and negative ion (ESI-) modes. The test instruments were the 2777C UPLC system (Waters, USA) for liquid chromatography and SYNAPT G2 XS QTOF (Waters, USA) for mass spectrum analysis.

Nitrogen was used as the dry gas and cone gas with the parameters described in Table S6. The separation of all of the samples (injection volume $10 \mu \mathrm{L}$ ) was performed on a ACQUITY UPLC BEH C18 column (Waters, USA) (dimension $100 \times 2.1 \mathrm{~mm}, 1.7 \mu \mathrm{m}$ particle size). Liquid Chromatographic column parameters with mobile phase A (water), mobile phase B (acetonitrile), and the speed $0.4 \mathrm{~mL} / \mathrm{min}$, and the gradient of mobile phase were $0 \sim 2 \min$ with $100 \% \mathrm{~A}-100 \% \mathrm{~A}$, $2 \sim 12$ min with $100 \%$ A-0\% A, 12 14 min with $0 \%$ A-0\% A, 14 15 min with $0 \%-100 \%$ A.

4.8. Data processing and statistics

Insect-infested samples and their corresponding control groups were prepared as described above. The MS original data was analyzed by Progenesis QI (ver. 2.2) software to obtain the peak $(\mathrm{mz})$ retention time and ion area. The normalized data were introduced to SIMCA-P V11.0 (Umetrics, Sweden) for PCA and for OPLS-DA analysis. We analyzed the QC samples with PCA, and their TIC (total ion current) map (Fig. S1). TIC map was used to determine the status of the instrument, the greater the overlap of the QC sample replicates, the more stable of the instrument. We then conducted univariate analysis by $t$ test and the $p$-value in the $t$ test after FDR correction then produced $q$-values. The results were considered to be significant when the $q$-value was less than 0.05 . OPLS-DA was carried out to investigate and visualize the pattern of metabolite changes. The differential metabolites were selected when the statistically significant threshold of VIP values obtained from the OPLS-DA model was larger than one. Log2 fold change (FC $\geq 1.2$ or $\leq 0.8$ ) was used to show how these selected differential metabolites varied between groups [32-34]. The related pathways of each metabolite were also listed by searching the KEGG pathway database (http://www.genome.jp/kegg/), and the metabolite molecular formula of matched metabolites was further identified by isotopic distribution measurement.

Metabolomics data have been deposited to the EMBL-EBI MetaboLights database with the identifier MTBLS573. The complete dataset can be accessed here : http://www.ebi.ac.uk/metabolights/MTBLS573 4.9. Data analysis

We used Microsoft Excel 15. 37, Mac Preview (8.1), Adobe Photoshop (2017.0.0) and Prims 6 to analyze the data and prepare the figures. We used the R package (Ver. 3.2.3). for heat map analysis. We used the online Venn Diagram Generator (http://www.pangloss.com/seidel/Protocols/venn.cgi) for Venn map analysis. 4.10. Real Time Quantitative PCR (RT-qPCR)

The plant samples were collected followed by the previously description. Total RNA from each sample was extracted using the RNAprep Pure Plant Kit (TIANGEN, China) according to the manufacturer's protocol. Approximately $1 \mu \mathrm{g}$ RNA was reverse transcribed to cDNA using a 
409 PrimeScriptTM RT reagent kit (perfect real time) (TaKaRa, Dalian, China) following the

410 manufacturer's protocol. The RT-qPCR was carried out using GoTaq Qpcr Master Mix (Promega,

411 USA) on an Eppendorf Mastercycler eprealplex 2.2 (Germany) with three biological replicates and

412 three technical replicates. The thermal cycle conditions used in the RT-qPCR were $95^{\circ} \mathrm{C}$ for $2 \mathrm{~min}$,

413 followed by 40 cycles of $95^{\circ} \mathrm{C}$ for $15 \mathrm{~s}$ and $60^{\circ} \mathrm{C}$ for $1 \mathrm{~min}$. The relative gene expression was

414 processed using the $2^{-} \Delta{ }^{\mathrm{Ct}}$ method [57], and two housekeeping genes [35](GhHis3 with GenBank

415 accession AF024716 and GhUBQ7 with GenBank accession DQ116441) were used in the RT-

416 qPCR analysis. The three hours control group was set as the reference sample for data

417 normalization. All of the primer pairs used for RT-qPCR were designed using the online

418 PrimerQuest Tool (http://sg.idtdna.com/PrimerQuest/Home/Index) and are listed in Table S6.

419 Differences in expression level were tested for significance by a one-way ANOVA with means

420 separation using Tukey's HSD (IBM SPSS Statistics Ver.22).

421

422

423

\section{Author Contributions}

HL, SZ, HX H and JJ C designed the research. HL performed the research. HL, JYL, CYW, LML, 425 LJ Z, XZ Z, LW and XKG contributed reagents/materials/analyses. HL and SZ wrote the paper 426 All authors reviewed the final manuscript.

\section{Acknowledgements}

431 Project support was provided by the National Natural Science Foundation of China (Grant No.

432 31572015) and the National Special Transgenic Project of China (Grant No.2016ZX08012-004).

433 We appreciated Bio information analysis engineer Qi Yin of BGI-Shenzhen for technical

434 assistance.

\section{Conflict of interest disclosure}

438 The authors declare no competing financial interests.

439

440 


\section{References}

1. Kessler, A.; Baldwin, I. T. Plant responses to insect herbivory: the emerging molecular analysis. Annual Review of Plant Biology. 2002, 53, 299-328.

2. Truong, D. H.; Heuskin, S.; Delaplace, P.; Francis, F.; Lognay, G. VOC emissions and protein expression mediated by the interactions between herbivorous insects and Arabidopsis plant. A review. Biotechnol. Agron. Soc. Environ. 2014, 18, 455-464.

3. Hanley, M. E.; Lamont, B. B.; Fairbanks, M. M.; Rafferty, C. M. Plant structural traits and their role in anti-herbivore defence. Perspectives in Plant Ecology, Evolution and Systematics. 2007, 8, 157-178.

4. $\mathrm{Wu}$, J.; Baldwin, L.T. New insights into plant responses to the attack from insect herbivores. Annual Review of Genetics. 2010, 44, 1-24.

5. Bergelson, J; Dwyer, G.; Emerson, J. J. Models and data on plant-enemy coevolution. Annual Review of Genetics. 2001, 35, 469-499.

6. Meaux, J. de; Mitchell-Olds, T. Evolution of plant resistance at the molecular level: ecological context of species interactions. Heredity. 2003, 91, 345-352.

7. Wu, K. M.; Lu, Y. H.; Feng, H. Q.; Jiang, Y. Y.; Zhao, J. Z. Suppression of cotton bollworm in multiple crops in China in areas with Bt toxin-containing cotton. Science. 2008, 321, 16761678.

8. Lu, Y. H.; Wu, K. M.; Jiang, Y.Y.; Xia, B.; Li, P.; Feng, H. Q.; Wyckhuys, K. A.G.; Guo, Y.Y. Mirid bug outbreaks in multiple crops correlated with wide-scale adoption of Bt cotton in China. Science. 2010, 328, 1151-1154.

9. Lu, Y. H.; Qiu, F.; Feng, H. Q.; Li, H. B.; Yang, Z. C.; Wyckhuys, K. A. G.; Wu, K. M. Species composition and seasonal abundance of pestiferous plant bugs (Hemiptera: Miridae) on Bt cotton in China. Crop. Prot. 2008, 27, 465-472.

10. Lu, Y. H.; Wu, K. M. Mirid bugs in China: pest status and management strategies. Outlooks on Pest Management. 2011, 22, 84-88.

11. Luo, J.; Liu, X. Y.; Liu, L.; Zhang, P. Y.; Chen, L. J.; Gao, Q.; Ma, W. H.; Chen, L. Z.; Lei, C. L. De novo analysis of the Adelphocoris suturalis Jakovlev metathoracic scent glands transcriptome and expression patterns of pheromone biosynthesis-related genes. Gene. 2014, $551,271-278$.

12. Theodoridis, G.; Gika, H. G.; Wilson, I. D. LC-MS-based methodology for global metabolite profiling in metabonomics / metabolomics. Trac-Trend. Anal. Chem. 2008, 27, 251-260.

13. Zhou, B., Xiao, J. F., Tuli, L.; Ressom, H. W. LC-MS-based metabolomics. Mol. Biosyst. 2012, 2, 470-481.

14. Xia, J; Sinelnikov, I. V.; Han, B.; Wishart, D. S. MetaboAnalyst 3.0-making metabolomics more meaningful. Nucleic Acids Res. 2015, 43, 251-257.

15. Zhu, H. J.; Wang, J. H.; Zhu, Z. J.; Johnson, C. H.; Patti, G.; Siuzdak, G. Liquid chromatography quadrupole time-of-flight mass spectrometry characterization of metabolites guided by the METLIN database. Nature Protocols. 2013, 8, 451-60.

16. Wu, W. W.; Wang, G.; Baek, S. J.; Shen, R. F. Comparative study of three proteomic quantitative methods, DIGE, cICAT, and iTRAQ, using 2D gel- or LC-MALDI TOF/TOF. $J$ Proteome Res. 2006, 5, 651- 658.

17. Li, Y.; Wang. X.; Hou, Y.; Zhou, X. Y.; Chen, Q. M.; Guo. C.; Xia, Q. Y.; Zhang, Y.; Zhao, P. Integrative proteomics and metabolomics analysis of insect larva brain: novel insights into the molecular mechanism of insect wandering behavior. J. Proteome Res. 2016, 15, 193-204. 
18. Huang, H. J.; Liu, C. W.; Huang, X. H.; Zhou, X.; Zhou, J. C.; Zhang, C. X.; Bao, Y. Y. Screening and functional analyses of Nilaparvata lugens salivary proteome. J. Proteome Res. 2016, 15, 1883-1896.

19. Keeling, C. I.; Li, M.; Sandhu, H. K.; Henderson, H.; Yuen, M. M. S.; Bohlmann, J. Quantitative metabolome, proteome and transcriptome analysis of midgut and fat body tissues in the mountain pine beetle, Dendroctonus ponderosae Hopkins, and insights into pheromone biosynthesis. Insect Biochemistry and Molecular Biology. 2016, 70, 170-183.

20. Tu, X. B.; Wang, J.; Hao, K.; Whitman, D. W.; Fan, Y. L.; Cao, G. C.; Zhang, Z. H. Transcriptomic and proteomic analysis of pre-diapause and non- diapause eggs of migratory locust, Locusta migratoria L. (Orthoptera: Acridoidea). Sci. Rep. 2015, 5, 1-13.

21. Chen, T. T.; Zhang, L.; Shang, H. H.; Liu, S. D.; Peng, J.; Gong, W. K; Shi, Y. Z.; Zhang, S. P.; Li, J. W.; Gong, J.W.; et al. iTRAQ-based quantitative proteomic analysis of cotton roots and leaves reveals pathways associated with salt stress. Plos One. 2016, 11, 1-15.

22. Liu, J.; Pang, C. Y.; Wei, H. L., Song, M. Z.; Meng, Y. Y.; Ma, J. H.; Fan, S. L.; Yu, S. X. iTRAQ-facilitated proteomic profiling of anthers from a photosensitive male sterile mutant and wild-type cotton (Gossypium hirsutum L.). Journal of Proteomics. 2015, 126, 68-81.

23. Radwanski, E. R.; Last, R. L. Tryptophan biosynthesis and biochemical and molecular genetics. The Plant Cell. 1995, 7, 921-934.

24. Wasternack, C. Jasmonates: An update on biosynthesis, signal transduction and action in plant stress response, growth and development. Annals of Botany. 2007, 100, 681-697,.

25. Wasternack, C.; Hause, B. Jasmonates: biosynthesis, perception, signal transduction and action in plant stress response, growth and development. An update to the 2007 review in Annals of Botany. Annals of Botany. 2013, 111, 1021-1058.

26. Cao, J. J.; Li, M. Y.; Chen, J.; Liu, P.; Li, Z. Effects of MeJA on Arabidopsis metabolome under endogenous JA deficiency. Sci. Rep. 2016, 6, 1-13.

27. Petricka, J. J.; Schauer, M. A.; Megraw, M.; Breakfield, N. W.; Thompson, J.W.; Georgiev, S.; Soderblom, E. J.; Ohler, U.; Moseley, M. A.; Grossniklaus, U.; et al. The protein expression landscape of the Arabidopsis root. Proc. Natl. Acad. Sci. U.S.A. 2012, 109, 6811-6818.

28. Walley, J. W.; Shen, Z. X.; Sartor. R.; Wu, K. J.; Osborn, J.; Smith, L. G.; Wu, K. J.; Urich, M. A.; Nery, J. R.; Schnable, J.C. et al. Integration of omic networks in a developmental atlas of maize. Science. 2016, 353, 814-818.

29. Walley, J. W., Shen Z. X., Sartor. R.; Wu, K. J.; Osborn, J.; Smith, L. G.; Briggs, S. P. Reconstruction of protein networks from an atlas of maize seed proteotypes. Proc. Natl. Acad. Sci. U.S.A. 2013, 110, E4808-E4817.

30. Zhu, M. M.; Dai, S. J.; McClung, S.; Yan, X. F.; Chen, S. X. Functional differentiation of Brassica napus guard cells and mesophyll cells revealed by comparative proteomics. Molecular \& Cellular Proteomics. 2009, 8, 752-766.

31. Wen, B.; Zhou, R.; Feng, Q.; Wang, Q. H.; Wang, J.; Liu, S. Q. IQuant: An automated pipeline for quantitative proteomics based upon isobaric tags Proteomics. 2014, 14, 2280 2285.

32. Chu, P.; Yan, G. X.; Yang, Q.; Zhai, L. N.; Zhang, C.; Zhang, F. Q.; Guan, R. Z. iTRAQbased quantitative proteomics analysis of Brassica napus leaves reveals pathways associated with chlorophyll deficiency. Journal of Proteomics. 2015, 113, 244-259.

33. Chen, Y. Z.; Pang, Q. Y.; He, Y.; Zhu, N.; Branstrom, I.; Yan, X. F.; Chen, S. X. Proteomics and metabolomics of Arabidopsis responses to perturbation of glucosinolate biosynthesis. Mol Plant. 2012, 5, 1138-50. 
34. Zhou, C. X.; Zhou, D. H.; Elsheikha, H. M.; Zhao, Y.; Suo, X.; Zhu, X. Q. Metabolomic profling of mice serum during toxoplasmosis progression using liquid chromatography-mass spectrometry. Sci. Rep. 2016, 6, 1-13.

35. Wang, M.; Wang, Q.; Zhang, B. Evaluation and selection of reliable reference genes for gene expression under abiotic stress in cotton (Gossypium hirsutum L.). Gene. 2013, 530, 44-50.

36. Turlings, T. C.; Tumlinsonm, J. H.; Lewis, W. J. Exploitation of herbivore-induced plant odors by host-seeking parasitic wasps. Science. 1990, 250, 1251-1253.

37. Kessler, A.; Baldwin, I. T. Plant responses to insect herbivory: the emerging molecular analysis. Annual Review of Plant Biology. 2002, 53, 299-328.

38. Bede, J. C.; Musser, R. O.; Felton, G. W; Korth, K. L. Caterpillar herbivory and salivary enzymes decrease transcript levels of Medicago truncatula genes encoding early enzymes in terpenoid biosynthesis. Plant Molecular Biology. 2006, 4, 519-531.

39. Wu, G.; Guo, J. Y.; Wan, F. H.; Xiao, N. W. Responses of three successive generations of beet armyworm, Spodoptera exigua, fed exclusively on different levels of gossypol in cotton leaves. Journal of Insect Science. 2010, 10, 1-11.

40. Aharoni, A.; Jongsma, M. A.; Bouwmeester, H. J. Volatile science? Metabolic engineering of terpenoids in plants. Trends in Plant Science. 2005, 10, 594-602.

41. Howe, G. A.; Jander, G. Plant immunity to insect herbivores. Annual Review of Plant Biology. 2008, 59, 41-66.

42. Du, L.; Ge, F.; Zhu, S.; Parajulee, M. N. Effect of cotton cultivar on development and reproduction of Aphis gossypii (Homoptera: Aphididae) and its predator Propylaea japonica (Coleoptera: Coccinellidae). Journal of Economic Entomology. 2004, 97, 1278-1283.

43. McAuslane, H. J.; Alborn, H. T. Systemic induction of allelochemicals in glanded and glandless isogenic cotton by Spodoptera exigua feeding. Journal of Chemical Ecology. 1998, 24, 399-416.

44. Townsend, B. J.; Poole, A.; Blake, C. J.; Llewellyn, D. J. Antisense suppression of a (+)-deltacadinene synthase gene in cotton prevents the induction of this defense response gene during bacterial blight infection but not its constitutive expression. Plant Physiology. 2005, 138, 516528.

45. Morawo, T.; Fadamiro, H. Duration of plant damage by host larvae affects attraction of two parasitoid species (Microplitis croceipes and Cotesia marginiventris) to cotton: implications for interspecific competition. Journal of Chemical Ecology. 2014, 40, 1176-1185.

46. Sanchez-Vallet, A.; Ramos, B.; Bednarek, P.; López, G.; Piślewska-Bednarek, M.; Schulzelefert, P.; Molina, A. Tryptophan-derived secondary metabolites in Arabidopsis thaliana confer non-host resistance to necrotrophic Plectosphaerella cucumerina fungi. The Plant Journal. 2010, 63, 115-127.

47. Grant, M. R.; Jones, J. D. Hormone (dis)harmony moulds plant health and disease. Science. 2009, 324, 750-752.

48. Feussner, I.; Wasternack, C. The lipoxygenase pathway. Annual Review of Plant Biology. 2002, 53, 275-297.

49. Ziegler, J.; Stenzel, I.; Hause, B.; Maucher, H.; Hamberg, M.; Grimm, R.; Ganal, M.; Wasternack, C. Molecular cloning of allene oxide cyclase: the enzyme establishing the stereochemistry of octadecanoids and jasmonates. Journal of Biological Chemistry. 2000, 275, 19132-19138.

50. Stintzi, A.; Weber, H.; Reymond, P.; Browse, J.; Farmer, E. E. Plant defense in the absence of jasmonic acid: the role of cyclopentenones. Proc Natl Acad Sci USA. 2001, 98, 12837-12842. 
51. Liu, Q. S.; Wang, X. Y.; Tzin, V.; Romeis, J.; Peng, Y. F.; Li, Y. H. Combined transcriptome and metabolome analyses to understand the dynamic responses of rice plants to attack by the rice stem borer Chilo suppressalis (Lepidoptera: Crambidae). BMC Plant Biology. 2016, 16, 1 17.

52. Alborn, H. T.; Turlings, T. C. J.; Jones, T. H.; Stenhagen, G.; Loughrin, J. H.; Tumlinson, J. H. An elicitor of plant volatiles from beet armyworm oral secretion. Science. 1997, 276, 945-949.

53. Lu, Y. H.; Wu, K. M.; Cai, X. M.; Liu, Y.Q. A rearing method for mirids using the green bean, Phaseolus vulgaris in the laboratory. Acta Phytophylacica Sinica. 2008, 35, 251-269.

54. Saravanan, R. S.; Rose, J. K. C. A critical evaluation of sample extraction techniques for enhanced proteomic analysis of recalcitrant plant tissues. Proteomics. 2004, 4, 2522-2532.

55. Kruger, N. J. The bradford method for protein quantitation. Basic Protein and Peptide Protocols. 2nd Edition. 1994, 15-21.

56. Vizcaino, J. A.; Cote, R. G.; Csordas, A.; Dianes, J. A.; Fabregat, A.; Forster, J. M.; Griss, J.; Alpi, E.; Birim, M.; Contell, J.; et al. The prteomics identifications (PRIDE) database and associated tools: status in 2013, Nucleic Acids Res. 2013, 41, D1063-1069.

57. Livak, K. J.; Schmittgen, T. D. Analysis of relative gene expression data using real-time quantitative PCR and the $2^{-\Delta \mathrm{Ct}}$. Methods. 2001, 25, 402-408.

Table 1. Pathway enrichment analysis of DEPs of P48E-VS-P48C

\begin{tabular}{|c|c|c|c|c|c|}
\hline & Pathway & DEPs & $q$-value & $\begin{array}{l}\text { Pathway } \\
\text { ID }\end{array}$ & $\begin{array}{l}\text { Primary /Secondary } \\
\text { Metabolites }\end{array}$ \\
\hline 1 & alpha-linolenic acid metabolism & 19 & 0.0001 & ko00592 & $\mathrm{b}$ \\
\hline 2 & $\begin{array}{l}\text { Fructose and mannose } \\
\text { metabolism }\end{array}$ & 26 & 0.0001 & ko00051 & $\mathrm{a}$ \\
\hline 3 & $\begin{array}{l}\text { Amino sugar and nucleotide } \\
\text { sugar metabolism }\end{array}$ & 42 & 0.0007 & ko00520 & $\mathrm{a}$ \\
\hline 4 & Selenocompound metabolism & 12 & 0.0009 & ko00450 & \\
\hline 5 & Protein digestion and absorption & 5 & 0.0013 & ko04974 & $\mathrm{a}$ \\
\hline 6 & $\begin{array}{l}\text { Biosynthesis of unsaturated fatty } \\
\text { acids }\end{array}$ & 11 & 0.0018 & ko01040 & a \\
\hline 7 & Biosynthesis of amino acids & 69 & 0.0023 & ko01230 & $\mathrm{a}$ \\
\hline 8 & $\begin{array}{l}\text { D-Glutamine and D-glutamate } \\
\text { metabolism }\end{array}$ & 4 & 0.0024 & ko00471 & a \\
\hline 9 & $\begin{array}{l}\text { Ascorbate and aldarate } \\
\text { metabolism }\end{array}$ & 23 & 0.0114 & ko00053 & \\
\hline 10 & beta-alanine metabolism & 13 & 0.0210 & ko00410 & $\mathrm{a}$ \\
\hline 11 & Glutathione metabolism & 23 & 0.0236 & ko00480 & $\mathrm{a}$ \\
\hline
\end{tabular}




$\begin{array}{llcccc}12 & \text { Cysteine and methionine } & 26 & 0.0288 & \text { ko00270 } & \text { a } \\ & \text { metabolism } & & & & \\ 13 & \text { Fatty acid degradation } & 15 & 0.0291 & \text { ko00071 } & \mathrm{a} \\ 14 & \text { Lysine degradation } & 8 & 0.0354 & \text { ko00310 } & \mathrm{a} \\ 15 & \text { Valine, leucine and isoleucine } & 17 & 0.0364 & \mathrm{k} 000280 & \mathrm{a} \\ & \text { degradation } & & & & \\ 16 & \text { Citrate cycle (TCA cycle) } & 19 & 0.0428 & \mathrm{k} 000020 & \mathrm{a}\end{array}$

\begin{tabular}{lllll}
\hline & Pathway & No. All $^{\text {a }}$ & Mode & Pathway ID \\
\hline 1 & Metabolic pathways & 269 & ESI+ & map01100 \\
2 & Biosynthesis of secondary metabolites & 213 & ESI+ & map01110 \\
3 & Sesquiterpenoid and triterpenoid biosynthesis & 40 & ESI+ & map00909 \\
4 & Tryptophan metabolism & 26 & ESI+ & map00380 \\
5 & Isoquinoline alkaloid biosynthesis & 22 & ESI+ & map00950 \\
& Tropane, piperidine and pyridine alkaloid & 20 & ESI+ & map00960 \\
6 & biosynthesis & & & \\
7 & Diterpenoid biosynthesis & 20 & ESI+ & map00904 \\
8 & Phenylpropanoid biosynthesis & 20 & ESI+ & map00940 \\
9 & Biosynthesis of amino acids & 18 & ESI+ & map01230 \\
10 & Amino sugar and nucleotide sugar metabolism & 18 & ESI+ & map00520 \\
11 & ABC transporters & 18 & ESI+ & map02010 \\
12 & Limonene and pinene degradation & 16 & ESI+ & map00903 \\
13 & alpha-linolenic acid metabolism & 16 & ESI+ & map00592 \\
14 & Monoterpenoid biosynthesis & 16 & ESI+ & map00902 \\
15 & Fructose and mannose metabolism & 16 & ESI+ & map00051 \\
16 & Phenylalanine metabolism & 15 & ESI+ & map00360 \\
\hline 17 & Metabolic pathways & 38 & ESI- & map01100 \\
18 & Biosynthesis of secondary metabolites & 37 & ESI- & map01110 \\
19 & alpha-linolenic acid metabolism & 10 & ESI- & map00592 \\
20 & Tryptophan metabolism & 8 & ESI- & map00380 \\
21 & Phenylpropanoid biosynthesis & map00940
\end{tabular}




$\begin{array}{lllll}22 & \text { Isoquinoline alkaloid biosynthesis } & 5 & \text { ESI- } & \text { map00950 } \\ 23 & \text { Porphyrin and chlorophyll metabolism } & 4 & \text { ESI- } & \text { map00860 } \\ 24 & \text { Phenylalanine metabolism } & 4 & \text { ESI- } & \text { map00360 } \\ 25 & \text { Biosynthesis of amino acids" } & 4 & \text { ESI- } & \text { map01230 } \\ 26 & \text { Cysteine and methionine metabolism } & 4 & \text { ESI- } & \text { map00270 } \\ 27 & \text { Flavonoid biosynthesis } & 4 & \text { ESI- } & \text { map00941 } \\ 28 & \text { Cutin, suberine and wax biosynthesis } & 3 & \text { ESI- } & \text { map00073 } \\ 29 & \text { Monoterpenoid biosynthesis } & 3 & \text { ESI- } & \text { map00902 } \\ 30 & \text { Glucosinolate biosynthesis } & 3 & \text { ESI- } & \text { map00966 }\end{array}$

605 a, the total number of differentially expressed on proteins

606 Table 3. Up/down regulated metabolite in Tryptophan metabolism pathway in cotton after

\begin{tabular}{llll}
\hline & Metabolite & Up/down-regulated & ESI+/ ESI-Mode \\
\hline 1 & 5-hydroxykynurenamine & down & ESI+ \\
2 & 5-hydroxykynurenine & down & ESI+ \\
3 & 5-hydroxy-L-tryptophan & down & ESI+ \\
4 & 6-hydroxymelatonin & down & ESI+ \\
5 & formyl-5-hydroxykynurenamine & down & ESI+ \\
6 & 2-formylaminobenzaldehyde & down & ESI+, ESI- \\
7 & N-formylkynurenine & down & ESI+ \\
8 & L-kynurenine & down & ESI+ \\
9 & 3-hydroxy-L-kynurenine & down & ESI+ \\
10 & 3-hydroxykynurenamine & down & ESI+ \\
11 & 2,3-dihydroxyindole & down & ESI+ \\
12 & indole-3-acetaldehyde & down & ESI+, ESI- \\
13 & indole-3-acetaldoxime & down & ESI+ \\
14 & indole-3-acetonitrile & down & ESI+, ESI- \\
15 & Indole-3-acetamide & down & ESI+, ESI- \\
16 & 3-methyldioxyindole & down & ESI+ \\
17 & 3-methoxyanthranilate & down & ESI+ \\
18 & Cinnavalininate & down & ESI+ \\
\hline 19 & tryptophan & down & ESI- \\
20 & 5-hydroxy-N-formylkynurenine & ESI- \\
& & &
\end{tabular}




\begin{tabular}{llll}
21 & melatonin & down & ESI- \\
\hline 22 & tryptamine & up & ESI+ \\
23 & 5 -(2'-carboxyethyl)-4,6-dihydroxypicolinate & up & ESI+ \\
24 & N-acetylserotonin & up & ESI+ \\
35 & Isophenoxazine & up & ESI+ \\
\hline 26 & 2-aminophenol & down & ESI+ \\
27 & 2-aminophenol & up & ESI-
\end{tabular}

609

610

\section{Figures legends}
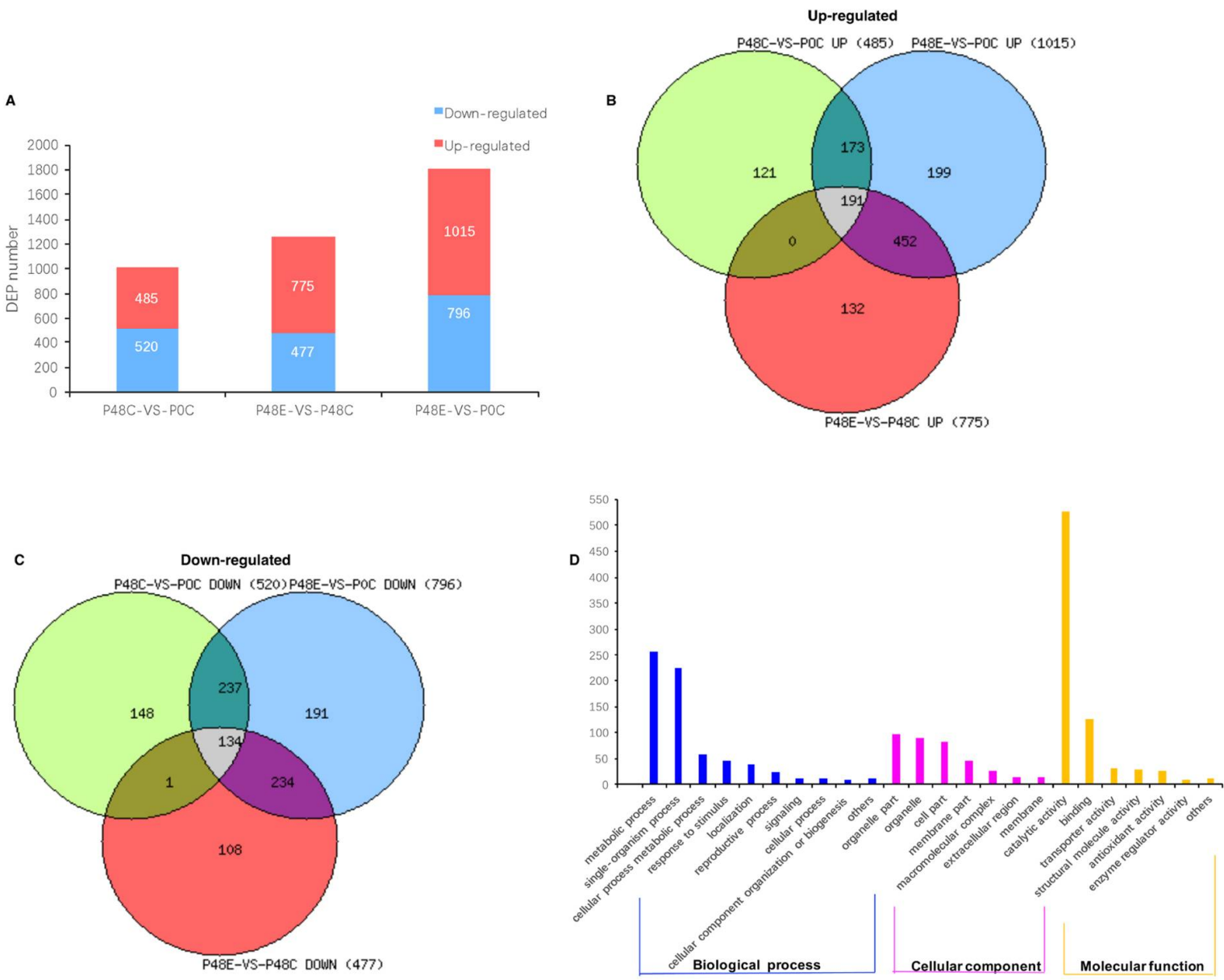

612 Fig 1. Information of identified proteins and GO enrichment analysis of DEPs. (A) Number

613 of up- and down-regulated proteins in different experimental groups: $\mathrm{P} 48 \mathrm{C}$ compared to P0C,

614 P48E compared to POC, and P48E compared to P48C. (P48E represents the plants that were

615 infested with insects for $48 \mathrm{~h}$. P48C represents the control plants that were not infested but simply

616 grown for $48 \mathrm{~h}$. P0C represents control plants that were the experimental cotton plants without 
617 insect infestation). (B) Venn diagram showing common or uniquely up-regulated proteins in 618 different experimental groups. (C) Venn diagram showing common or uniquely down-regulated 619 proteins in different experimental groups. (D) GO annotation and functional classification of the 620 differentially expressed proteins in cotton plants.
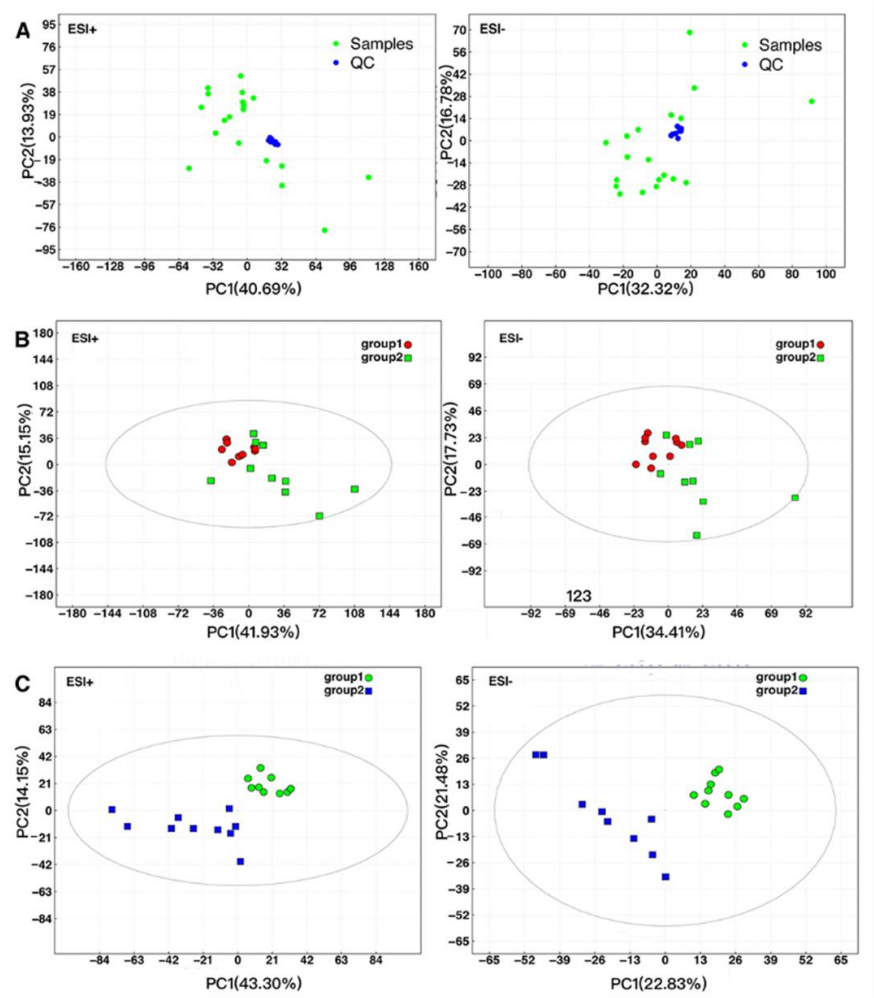

622 Fig 2. PCA and OPLS-DA score plot. (A) PCA of QC sample. (B) PCA score plot of $A$.

623 suturalis infected and control groups. (C) OPLS-DA score plot of $A$. suturalis infected and control 624 groups (with $\mathrm{R}^{2}=0.861, \mathrm{Q}^{2}=0.626$ in positive ion mode, and $\mathrm{R}^{2}=0.904, \mathrm{Q}^{2}=0.690$ in negative ion 625 mode). Group 1 represents the plants that were infested with insects for $48 \mathrm{~h}$, group 2 represents 626 the plant that were not infested but simply grown for $48 \mathrm{~h}$. ESI+ represents positive ion mode, 627 ESI- represent negative ion mode. 
628
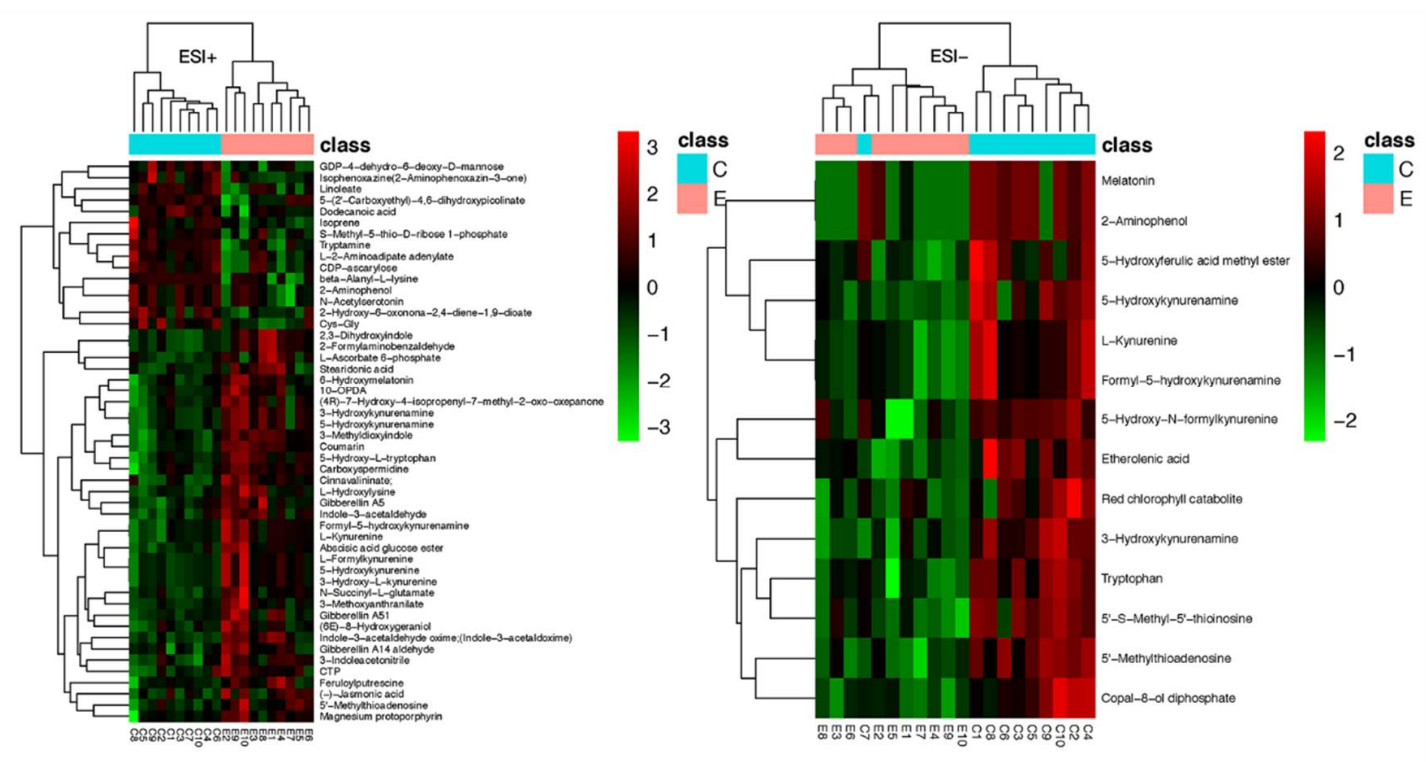

Fig 3. Heat maps comparison of metabolites of the $A$. suturalis infected group and control group. Heat maps represents the highly significantly differential variables between the insect infected groups and the corresponding control groups using R package (Ver. 3.2.3). ESI+ represents positive ion mode, ESI- represents negative ion mode. E represents the insect infected group, $\mathrm{C}$ represents the corresponding control group.

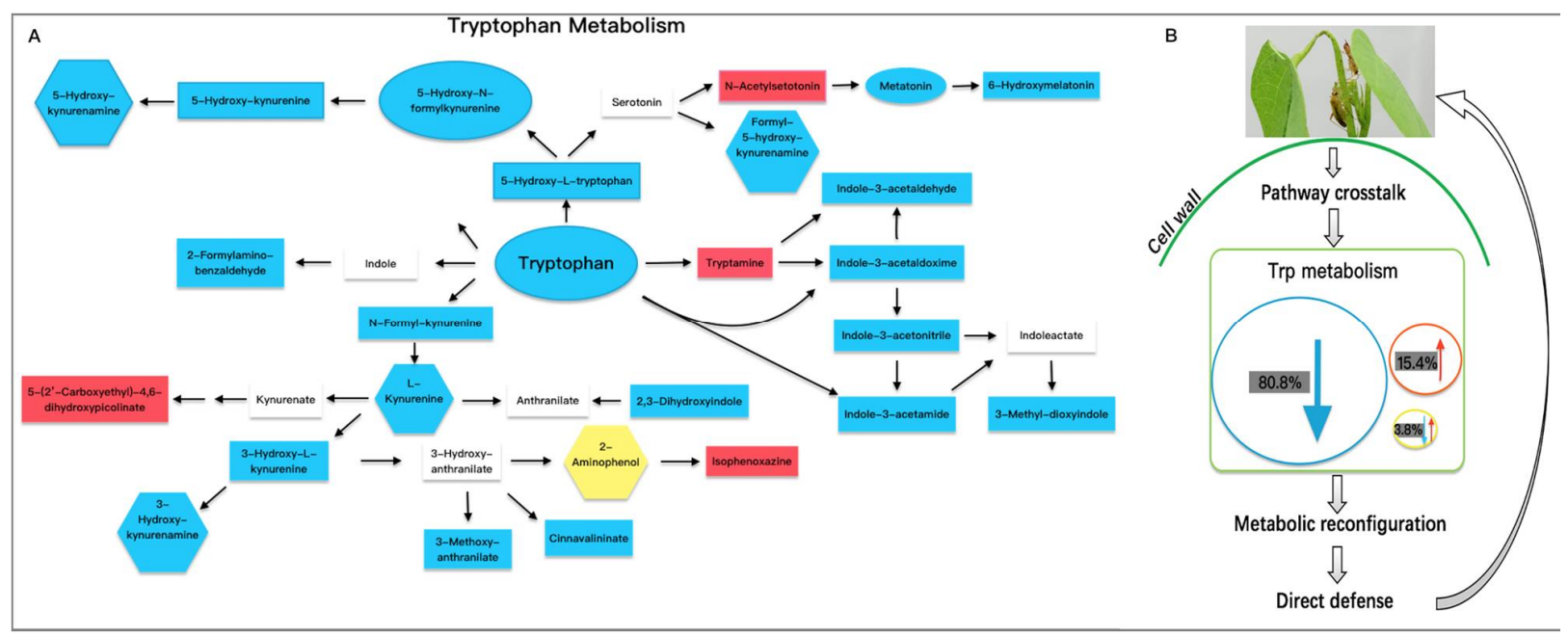

Fig 4. Tryptophan metabolism pathway analysis. (A) Metabolites changed in tryptophan metabolism pathway. Red represents upregulated, blue represents downregulated, yellow represents upregulated in positive mode, down regulated in negative mode, white represents unchanged metabolites in both ion mode. Rectangular box represents metabolites changed in positive ion mode, elliptic box represents metabolites changed negative ion mode, regular hexagon box represents changed metabolites in both ion mode. (B) A schematic representation showing how the metabolites in tryptophan metabolism pathway affect plant defense attacked by insect. (The photo was taken by the author Hui $\mathrm{Lu}$ ) 


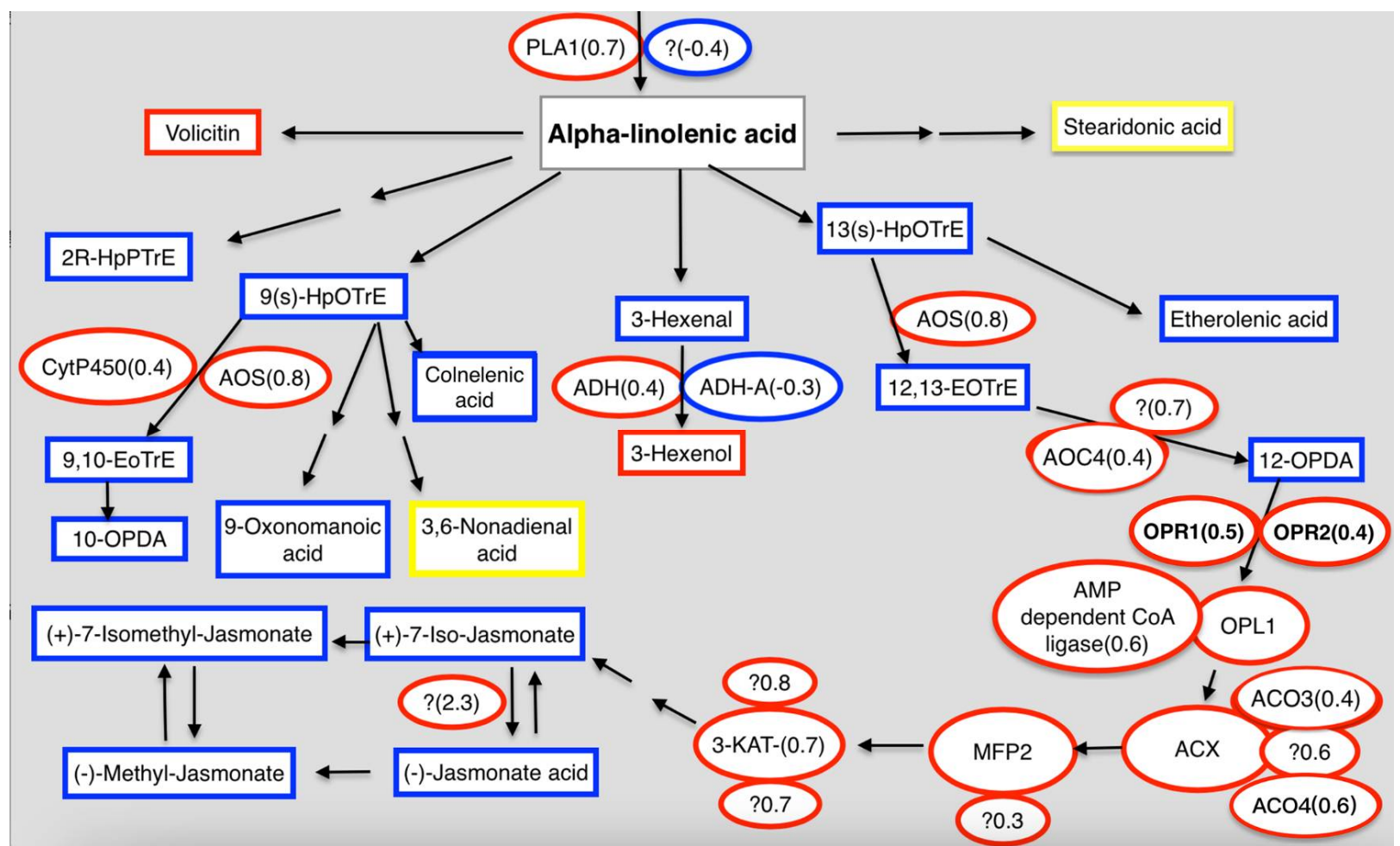

Fig 5. Integrated proteomics and metabolomics analysis alpha-linolenic acid metabolism pathway. Rectangular box represents the changed metabolites, elliptic box represents the changed proteins (the number represent the log2 fold change, "?" represents the unknown or predicted protein), red represents upregulated, blue represents downregulated, yellow represents up and downregulated, gray represents unchanged metabolites. 

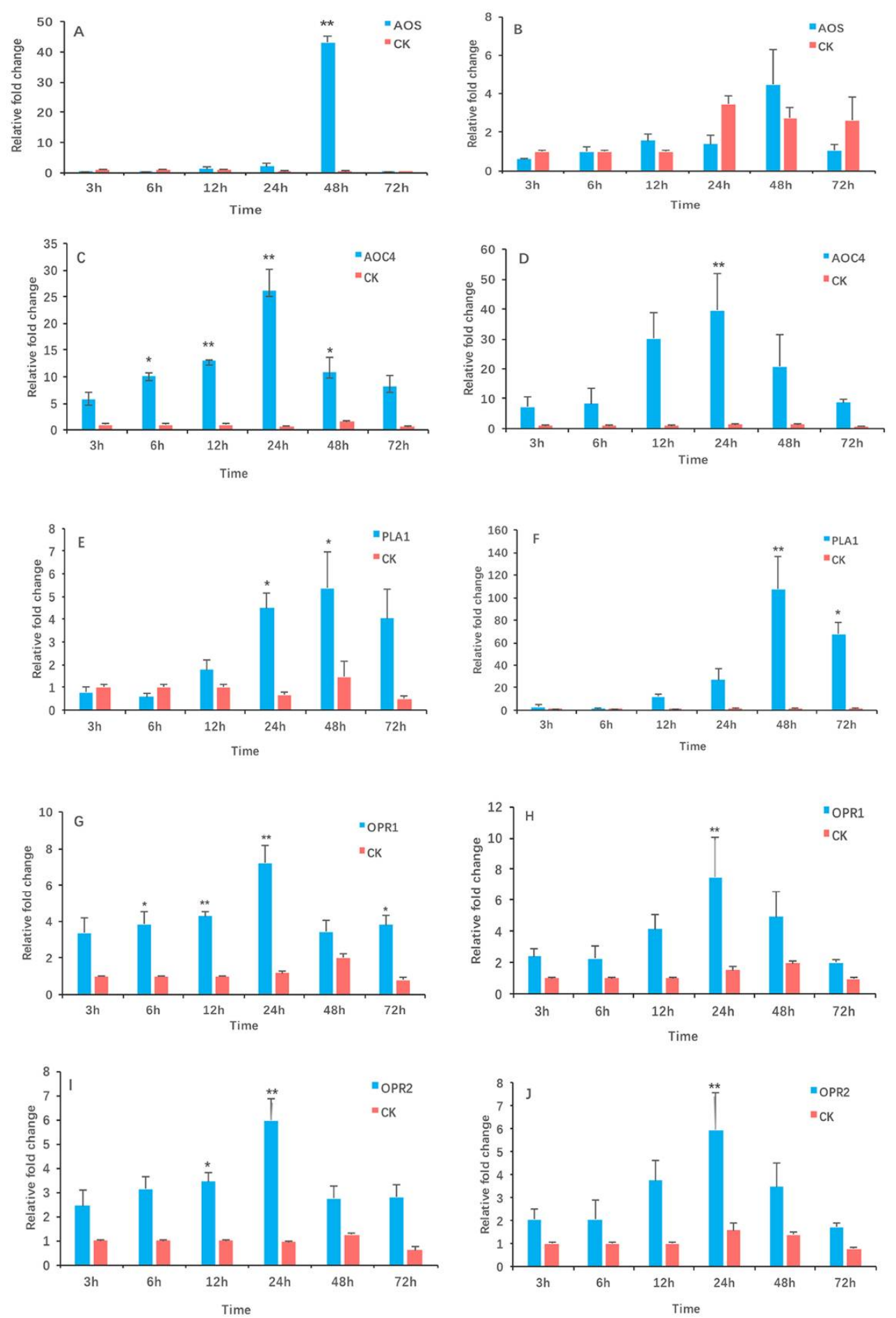

651 Fig 6. The RT-qPCR analysis of proteins related to the alpha-linolenic acid metabolism

652 pathway. Cotton samples were collected as previously described, and total RNA was extracted for 653 RT-qPCR analysis. A, C, E, G, I represent relative expression of different genes in cotton main 654 leaf, respectively. B, D, F, H, J represent relative expression of different genes in cotton 
655 cotyledon, respectively. AOS: allene oxide synthase (CotAD_35840), PLA1: phospholipase A1656 llgamma-like (CotAD_52791), AOC4: allene oxide cyclase 4 (Cotton_D_gene_10007844), OPR1: 657 12-oxophytodienoate reductase 1 (CotAD_59461), OPR2: 12-oxophytodienoate reductase 2 658 (Cotton_D_gene_10037325). The GhHis3 and GhUBQ7 gene were used as the reference gene.

659 Three biological replicates were performed. The three hours control group was set as the reference 660 sample for data normalization. Significant differences between treatments and their corresponding 661 control groups were identified by a one-way ANOVA with means separated using Tukey's HSD, 662 and two levels (“*” $p<0.05$ and “**” $p<0.01$ ) were adopted to judge the significance of 663 difference. 